\title{
Computer printed denture labeling: A simplified technique
}

\author{
Adhikari A ${ }^{1}$, Mathema SRB ${ }^{2}$, Maksey B ${ }^{3}$, Maharjan $\mathrm{SK}^{4}$, Dhyako N5 , Gurung A ${ }^{6}$, Pandey B , \\ Subedi $\mathrm{S}^{8}$ \\ $1,5,6,7,8 \mathrm{PG}$ Resident, ${ }^{2}$ Professor, ${ }^{3,4}$ Associate Professor, Department of Prosthodontics and Maxillofacial Prosthetics, \\ Peoples Dental College and Hospital, Kathmandu, Nepal
}

\begin{abstract}
Importance and benefits of denture labeling have been widely described in forensic and dental community. It helps in identification and prevents the probability of losing dentures in geriatric edentulous patient. Several methods have been described in literature for the purpose of labeling denture. The article fully describes an inclusive method which is simple, economical, permanent and is done with equipment readily available in a laboratory.
\end{abstract}

Key words: Denture labeling; Denture; Edentulous; Forensic science; Inclusion method

\section{Introduction}

$\mathrm{H}$ uman teeth are unique structure that helps in personal identification of living or deceased person. In case of edentulous patients, loss of teeth results in significant bone loss raising inconsistencies in forensic analysis. ${ }^{1}$ Thus denture labeling in these patients provides personal identification of an individual for medico-legal purpose. Routine practice of denture labeling is encouraged by international dental associations and forensic odontologists. ${ }^{2,3}$ It can be of great value in hospitalized patients, unconscious patient, patients staying at elderly care center where switching of dentures may occur, patient with dementia or people involved in natural disasters.

To provide denture identification various labeling techniques are used in clinical practice. ${ }^{4,5}$ They are broadly classified into "surface markings" and "inclusion markings". Surface marking methods are less technique sensitive, easy to apply and cost effective. However they can cause tissue irritation from uneven surfaces, act as a medium for plaque retention compromising denture health and fades away with time requiring repeated application. ${ }^{6}$
Inclusion marking methods incorporates the label within the denture, making it more durable, maintains the integrity of polished surface and does not compromise denture health yet the incorporation of label within the denture might reduce the denture strength, can be expensive, requires various instruments, is time consuming and requires expertise. ${ }^{6}$

Here, we describe an inclusion marking technique of a processed denture for denture labeling which we used in our patient that was quick, easy and economic.

\section{Technique}

a. After the denture try in is complete, follow the regular process of denture fabrication.

b. Trim and sand paper the processed upper and lower denture maintaining a thickness of $2 \mathrm{~mm}$ overall.

c. Type the patients name in font 8 in Times New Roman format and print it in the rough side of the tracing paper.

d. Cut the printed part neatly and measure the length of cut section with a scale which came out to be $2 \mathrm{~cm}$ (Figure 1). 
e. Then create a trough with length of $2 \mathrm{~cm}$ and depth of $1 \mathrm{~mm}$ in the disto-palatal side of the upper denture (Figure 2) and on the lingual side of the lower denture on the cameo surface (Figure 3 ) by trimming with a round acrylic trimming bur.

f. Sandwich the printed part in between a thin film of cyanoacrylate in the trough to position the label (Figure 4) and enclose this with autopolymerising clear acrylic resin (Figure 5).

g. The denture is then placed in pressure pot for 20 psi for curing of the residual monomer after which it is allowed to sit for 24 hours before denture delivery (Figure 6).

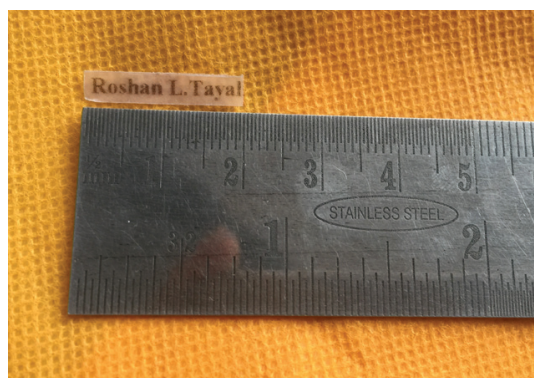

Figure 1

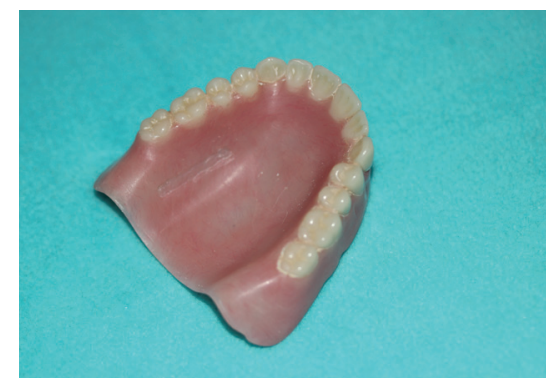

Figure 2

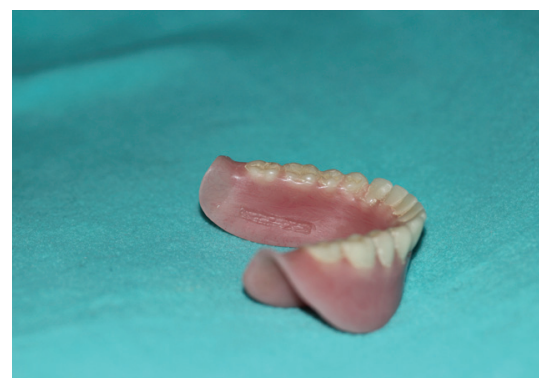

Figure 3
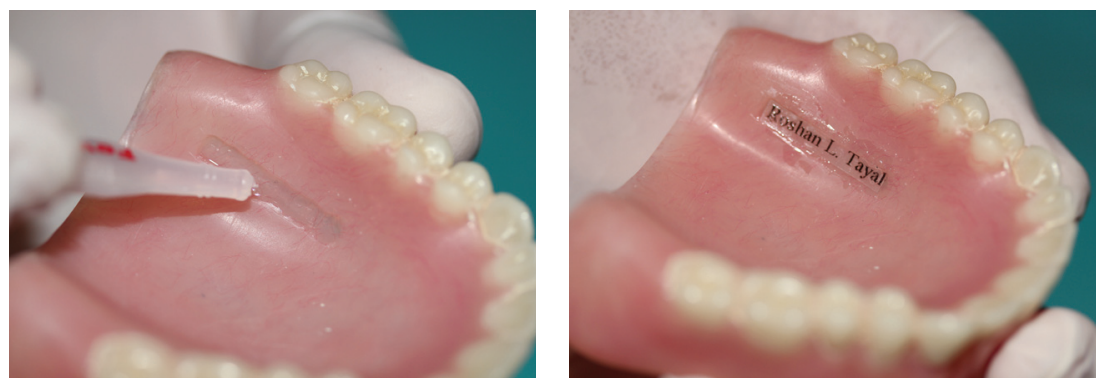

Figure 4

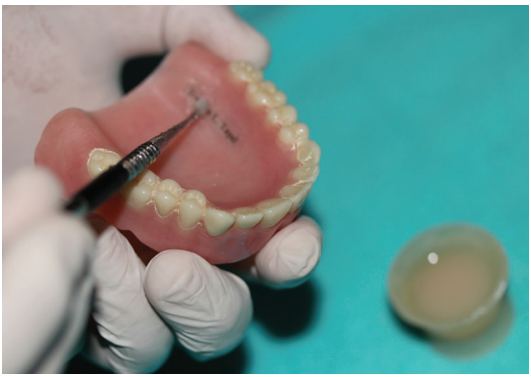

Figure 5
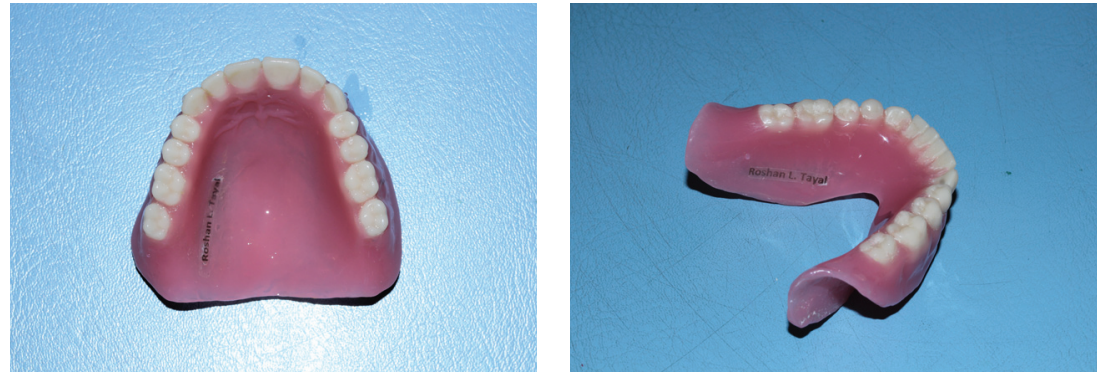

Figure 6 


\section{Discussion}

Denture labeling has various advantages and thus is advocated by forensic odontologists and validated by various international dental association. ${ }^{7}$ Unfortunately routine practice of denture labeling is not widely used in clinical practice because of misconception of the procedure being time consuming, expensive, lack of importance of denture labeling and use of multiple resources. Benefits of denture labeling reduces the problems to be caused by lost denture such as impact on patients quality of life, cost of fabricating a new denture and difficulty in adapting to the new dentures.

Our technique was an easy, economic and quick which can be used in resource limited setup as well. We used a digitally printed computer generated label in a tracing paper which is readily available. Since the label was printed only in a small part of this tracing paper it can be later utilized multiple times for printing new labels. The trough for placing the label was made on the cameo surface of palatal portion of maxillary and lingual of mandibular complete denture in order to avoid compromise in speech, function and esthetic of the patient. Cyanoacrylate adhesive used here had dual purpose. Firstly, it helped in stabilizing of the label. Secondly, it acted as a protective layer preventing the direct contact of monomer to the printed name. The technique described here is a post fabrication inclusion technique which gives benefit of being less technique sensitive and allows for accurate positioning of the label. Besides, patient approval can be taken before placing the label.

However, following are the limitation of this technique:

- The label used may not withstand thermal insult.
- Due to the use of clear autopolymerising resin, release of uncured monomer may occur and the denture may not be color stable in a long run.

\section{Conclusion}

Denture labeling is of great value when verifying a person's true identity. Efforts should be made to incorporate denture labeling understanding its benefit over the consequences of lost denture. Thus, Prosthodontics being aware of the standards and importance of denture labeling can play significant role by instructing and exposing the dental laboratories to place the denture labels in routine practice.

\section{References}

1. Richmond R, Pretty IA. Identification of the edentulous individual: An investigation into the accuracy of radiographic identifications. Journal of forensic sciences. $2010 \mathrm{Jul} ; 55(4): 984-7$.

2. McGivney GP. Marking of removable dental prosthesis. In: Averill DC, editor. Manual of forensic odontology. Colorado Springs: American Academy of Forensic Sciences; 1991. p. 62-6.

3. Thomas CJ, Mori T, Miyakawa O, Chung HG. In search of a suitable denture marker. The Journal of forensic odonto-stomatology. 1995 Jun;13(1):9-13.

4. Ling BC. Computer-printer denture microlabeling system. Journal of Prosthetic Dentistry. 1998 Mar 1;79(3):363-4.

5. Ibrahim WM. Denture microlabeling technique. Journal of Prosthetic Dentistry. 1996 Jul 1;76(1):104.

6. Richmond R, Pretty IA. Contemporary methods of labeling dental prostheses-A review of the literature. Journal of forensic sciences. 2006 Sep;51(5):1120-6.

7. Alexander PM, Taylor JA, Szuster FS, Brown KA. An assessment of attitudes to, and extent of, the practice of denture marking in South Australia. Australian dental journal. 1998 Oct;43(5):337-41. 